\title{
Caracterización de las infecciones respiratorias en pacientes adultos oncológicos
}

\author{
GINO FUENTES L.****, CARMEN VENEGAS G.*, MARCOS ORTEGA G.*, CATALINA BRICEÑO V.*, \\ JORGE DREYSE D.*, RICARDO RABAGLIATI B.** y FERNANDO SALDÍAS P.*
}

\section{Clinical characteristics of respiratory infections in patients with cancer}

Introduction: Infections are common in oncology. Aim: To characterize respiratory infections in hospitalized adult cancer patients. Methods: Prospective, descriptive study of febrile adult patients in oncology at the Hospital Clínico Universidad Católica de Chile, between April 2008 and April 2009. Results: 187 episodes were evaluated. Clinical infection was recognized in $70 \%$ and 33 episodes (25\%) were respiratory focus. Neoplastic disease corresponded to solid organ cancer in $77 \%$ of cases and 33\% were admitted with febrile neutropenia. The upper respiratory tract infection accounted for $36 \%$ and low respiratory tract infection $65 \%$ of cases; $55 \%$ was pneumonia. Etiology of these was isolated in 39\% (S. pneumoniae 2 cases, S. bovis 1, E. faecalis 1, P. jirovecii 2, S. maltophilia 1 and A. fumigatus 1). Hospital mortality for pneumonia was $22 \%$ and overall mortality $12 \%$. Conclusions: Respiratory infections are a major focus in adult cancer patients, highlighting pneumonia. It carries high mortality and varied etiology.

Key words: Infection, lung cancer, pneumonia, febrile neutropenia, oncology.

\section{Resumen}

Introducción: Las infecciones en oncología son frecuentes. Objetivo: Caracterizar las infecciones respiratorias en pacientes oncológicos hospitalizados. Métodos: Estudio descriptivo prospectivo de pacientes adultos oncológicos febriles en el Hospital Clínico de la Universidad Católica, entre abril de 2008 y abril de 2009. Resultados: Se evaluaron 187 episodios. Se identificó foco clínico en $70 \%$ y 33 episodios (25\%) correspondieron a foco respiratorio. La patología oncológica correspondió a tumores de órgano sólido en $77 \%$ de los casos y el 33\% ingresó con neutropenia febril. La infección respiratoria alta representó el 36\% y baja un 65\% de los casos; 55\% fueron neumonía. De estos, se aisló el agente causal en un 39\% (S. pneumoniae 2 casos, S. bovis 1, E. faecalis 1, P. jirovecii 2, S. maltophilia 1 y A. fumigatus 1). La letalidad en el hospital por neumonia fue $22 \%$ y la mortalidad general $12 \%$. Conclusiones: La infección respiratoria es un motivo de consulta y hospitalización frecuente en pacientes oncológicos, destacando la neumonía. Conlleva elevada mortalidad y etiología variada.

Palabras clave: Infección, Cáncer pulmonar, neumonía, neutropenia febril, oncología.

\section{Introducción}

Las infecciones en los pacientes con cáncer, son una importante causa de morbilidad y mortalidad $^{1-3}$. La investigación clínica ha estado dirigida principalmente al estudio de los pacientes neutropénicos. Sin embargo, existe un creciente número de pacientes en terapia oncológica que no presentan neutropenia pero desarrollan infecciones que requieren hospitalización. Tanto en pacientes con neutropenia febril y pacientes oncológicos no neutropénicos destacan las infecciones respiratorias, las cuales se asocian a una alta morbilidad y mortalidad ${ }^{4}$.

Financiamiento: Merck Sharp \& Dohme.

* Departamento de Enfermedades Respiratorias, Facultad de Medicina, Pontificia Universidad Católica de Chile.

** Departamento de Enfermedades Infecciosas, Facultad de Medicina, Pontificia Universidad Católica de Chile.

***Instituto de Medicina. Facultad de Medicina, Universidad Austral de Chile. 
Por otro lado, el aumento en la incidencia de infecciones en el ambiente hospitalario hace necesario implementar estrategias de vigilancia en los pacientes oncológicos. Además, el uso de esquemas antibióticos empíricos de amplio espectro y la emergencia de resistencia a antimicrobianos, hacen aún más necesaria esta vigilancia ${ }^{3-4}$.

En esta perspectiva, el objetivo de este trabajo es caracterizar las infecciones respiratorias en pacientes oncológicos que requieren hospitalización en nuestro centro.

\section{Materiales y Métodos}

El Hospital Clínico de la Pontificia Universidad Católica de Chile (HCUC) es un recinto hospitalario de nivel terciario de atención, ubicado en la ciudad de Santiago, Chile, donde anualmente se hospitalizan alrededor de 25.000 pacientes; cuenta con 493 camas de las cuales 58 corresponden preferentemente a cuidados de pacientes oncológicos adultos. Junto al HCUC existe un centro ambulatorio de diagnóstico y terapia del cáncer "Nuestra Señora de la Esperanza" al que anualmente consultan alrededor de 9.000 pacientes para evaluación o terapia oncológica, administrándose por año 1.800 ciclos de quimioterapia ambulatorios y 1.300 ciclos hospitalizados en HCUC donde también son referidos para procedimientos diagnósticos o terapéuticos, incluyendo manejo de patologías infecciosas.

Se diseñó este estudio prospectivo de vigilancia de infecciones en pacientes con cáncer hospitalizados en el HCUC, entre Abril de 2008 y Abril de 2009. Los criterios de inclusión fueron pacientes de ambos sexos mayores o iguales a 15 años, con cáncer hematológico o de órganos sólidos en quimioterapia y/o radioterapia, cursando con evento sospechoso de infección (fiebre o foco clínico de infección, con o sin Síndrome de Respuesta Inflamatoria Sistémica). Se diferenció a los pacientes en dos categorías: Neutropénicos y No Neutropénicos (Recuento absoluto de Neutrófilos RAN $\leq$ o $>500$ células/ $\mathrm{mm}^{3}$ ). Además se confeccionó una ficha para registro de datos de cada paciente, en la que se consignaron antecedentes biodemográficos de edad y género, enfermedad oncológica, etapa clínica y tratamiento (cirugía, quimioterapia y radioterapia), comorbilidades, score de MASCC (Multinational Association for Supportive Care in Cancer Risk Index) para pacientes neutropénicos, RAN, Leucocitos (Valor normal 4.500-11.000 células $/ \mathrm{mm}^{3}$ ), Proteína C Reactiva (PCR, valor normal $0-1 \mathrm{mg} / \mathrm{dL}$ ) y resultados de la evalua- ción clínico-microbiológica, considerando foco clínico de infección, hallazgos de los estudios imagenológicos, resultados de los cultivos microbiológicos (hemocultivos, urocultivo y otros), según les fuese solicitado a criterio de su médico tratante (cultivo de secreción bronquial o lavado broncoalveolar, estudio de virus respiratorios, serologías de microorganismos atípicos, baciloscopías, cultivos de Koch y hongos, etc), informe de susceptibilidad in vitro en aquellos casos con cultivos positivos, tratamientos antimicrobianos recibidos y condición de egreso.

Los episodios fueron catalogados como "infección clínicamente documentada" si se evidenciaban elementos clínicos y/o estudio de imágenes con focalización; "infección microbiológicamente documentada" si se demostraba microorganismo a través de cultivos u otro método diagnóstico y "fiebre de origen desconocido" (FOD) en caso que ni las manifestaciones clínicas, ni el estudio de imágenes ni microbiológico permitiera explicar el origen de la fiebre ${ }^{4-5}$.

Se procedió a realizar el análisis de los pacientes con foco clínico respiratorio, correspondiente a pacientes con síntomas y/o signos clínicos respiratorios altos (congestión nasal, rinorrea, odinofagia, descarga nasal posterior, disfonía) o bajos (tos, expectoración, dolor torácico, disnea, signos de focalización pulmonar) como también a pacientes con hallazgos radiológicos compatibles. En esta clasificación, la bronquitis, neumonía y empiema fueron definidas como infección respiratoria baja $\mathrm{a}^{4-5}$.

Frente a la identificación de Staphylococcus coagulasa negativa ( $S \mathrm{CoN}$ ), a modo de no incluir hemocultivos contaminados, se aplicaron los siguientes criterios de exclusión: cultivos positivos después de $27 \mathrm{~h}$ de incubación, un cultivo positivo con otro concomitante negativo o dos especies diferentes en dos hemocultivos positivos simultáneos ${ }^{6}$. En caso que se hubiese tomado sólo una muestra de hemocultivo con resultado positivo a SCoN se consideraba la opinión del médico tratante según si decidía incluir su cobertura en el esquema antimicrobiano.

\section{Análisis estadístico}

Se construyó una base de datos y se realizó el análisis estadístico con el programa Excel 2008. Los resultados de las variables nominales se expresan en porcentajes y las variables numéricas en promedio \pm desviación estándar (DS) e intervalo de confianza (IC). En el análisis comparativo de los pacientes se empleó el test de t-Student para las variables continuas y para las variables discontinuas test de $\chi^{2}$ o test de Fischer. 
Se consideró como diferencia estadísticamente significativa el valor de p menor o igual a 0,05 .

\section{Ética y financiamiento}

El protocolo fue aprobado por el Comité de Ética en Investigación de la Pontificia Universidad Católica de Chile. A todo paciente se le solicitó el consentimiento informado para participar en esta investigación.

El financiamiento de este estudio fue aportado por Merck Sharp \& Dohme, quienes no participaron en la recolección de los datos, análisis ni publicación de los mismos.

\section{Resultados}

Se evaluaron 187 episodios infecciosos en 146 pacientes adultos oncológicos. La mayor proporción correspondió a pacientes no neutropénicos $(65,8 \%)$. Dentro del grupo de pacientes neutro- pénicos, el RAN promedio fue $142,3 \pm 182,3$ células $/ \mathrm{mm}^{3} ; 66 \%$ presentaba RAN menor de 100 células $/ \mathrm{mm}^{3}$ y $34 \%$ entre 101 y 500 células/ $\mathrm{mm}^{3}$. En cuanto a la evaluación de riesgo de la neutropenia febril, el score de MASCC promedio correspondió a $20,5 \pm 4,2$, siendo el $42,3 \%$ pacientes de alto riesgo. El 24,1\% de los pacientes tenían indicación de antibióticos profilácticos, correspondiendo al $38 \%$ de los pacientes neutropénicos y $16 \%$ de los no neutropénicos $(\mathrm{p}<0,05)$ Las características generales de los pacientes incluidos en el protocolo se muestran en la Tabla 1.

Se identificó el foco clínico en 124 casos (66\%): Foco gastrointestinal $47 \%$, piel y partes blandas $13 \%$, urinario $12 \%$, sitio inserción catéter venoso central $2 \%$. En la Tabla 2 se presenta su diferenciación entre pacientes neutropénicos y no neutropénicos. Treinta y tres episodios $(26 \%)$ correspondieron a foco clínico respiratorio (12 infección respiratoria alta y 21 respiratoria baja). En este grupo, la edad media era 60 años \pm 15

Tabla 1. Características generales y comparación entre pacientes oncológicos febriles y pacientes oncológicos con infección respiratoria clínicamente documentada

\begin{tabular}{|c|c|c|c|}
\hline Características generales & $\begin{array}{l}\text { Foco infeccioso } \\
n=187 \text { episodios }\end{array}$ & $\begin{array}{l}\text { Foco clínico respiratorio } \\
\mathbf{n}=\mathbf{3 3} \text { episodios }\end{array}$ & $\mathbf{p}$ \\
\hline $\begin{array}{l}\text { Análisis } \\
\text { Neutropénicos } \\
\text { No neutropénicos }\end{array}$ & $\begin{array}{l}187 \text { episodios (146 pacientes) } \\
64(34,2 \%) \\
123(65,8 \%)\end{array}$ & $\begin{array}{l}33 \text { episodios (32 pacientes) } \\
11(33,2 \%) \\
22(66,7 \%)\end{array}$ & NS \\
\hline Edad (mediana años) & 53 años (15-91) & 58 años (15-89) & NS \\
\hline Género femenino $(\%)$ & 50,3 & 36,4 & NS \\
\hline $\begin{array}{l}\text { Lugar hospitalización inicial } \\
\text { - Baja complejidad (\%) } \\
\text { - Alta/mediana complejidad (UPC) (\%) }\end{array}$ & $\begin{array}{r}95,2 \\
4,8\end{array}$ & $\begin{array}{l}81,8 \\
18,2\end{array}$ & 0,001 \\
\hline $\begin{array}{l}\text { Cáncer hematológico }(\%) \\
\text { - Leucemia }(\%) \\
\text { - Linfoma }(\%) \\
\text { - Mieloma }(\%) \\
\text { Cáncer de órganos sólidos }(\%)\end{array}$ & $\begin{array}{l}33,7 \\
49,2 \\
39,7 \\
11,1 \\
66,3\end{array}$ & $\begin{array}{r}33,3 \\
18,2 \\
72,7 \\
9,1 \\
66,7\end{array}$ & NS \\
\hline
\end{tabular}

Tabla 2. Focos clínicos identificados en pacientes oncológicos febriles. Análisis comparativo entre pacientes neutropénicos y no neutropénicos

\begin{tabular}{|c|c|c|c|}
\hline Foco infeccioso & $\begin{array}{c}\text { Pacientes neutropénicos } \\
(\%)\end{array}$ & $\begin{array}{c}\text { Pacientes no neutropénicos } \\
(\%)\end{array}$ & $\mathbf{p}$ \\
\hline Clínica documentada & 65,6 & 71,5 & NS \\
\hline Gastrointestinal & 60,5 & 35,9 & $<0,05$ \\
\hline Infección Respiratoria Alta & 16,3 & 10,9 & NS \\
\hline Infección Respiratoria Baja & 9,3 & 19,6 & NS \\
\hline Piel y partes blandas & 9,3 & 14,1 & NS \\
\hline Urinario & 0 & 17,4 & $<0,05$ \\
\hline Fiebre de origen desconocido & 23,4 & 18,7 & NS \\
\hline
\end{tabular}


[rango: 15-89 años]. La enfermedad oncológica de base correspondió a enfermedad hematológica en 33\% de los casos (2 Leucemias, 8 Linfomas, 1 Mieloma Múltiple) y tumor de órgano sólido en 67\% (45\% Pulmón, 14\% Mama, 9\% Colon, $9 \%$ Ovario). El 94\% de los pacientes se presentó febril, $21 \%$ con leucocitosis $(19.286 \pm 4.571$ células $/ \mathrm{mm}^{3}$ [12.500-24.700]) y 94\% tenía PCR sérica elevada (13,6 $\pm 11 \mathrm{mg} / \mathrm{dL}[1,3-46])$. El 82\% de los pacientes ingresó a sala y $18 \%$ a Unidad de Pacientes Críticos (UPC). Un tercio de los pacientes ingresó con neutropenia febril (Tabla 1).

La infección respiratoria alta representó el 36\% de los casos y baja (bronquitis/neumonía/empiema) el 64\%. De los 21 casos de infecciones respiratorias bajas, 18 correspondieron a neumonía, 2 a bronquitis y uno a empiema (Figura 1). De las neumoníaas, se aisló el agente causal en 7 casos (39\%), correspondiendo a S. pneumoniae (2 casos identificados por hemocultivo), S. bovis (1, hemocultivo), E. faecalis (1, cultivo expectoración), $P$. jirovecii (2, PCR en gárgaras) y en un paciente S. maltophilia y Aspergillus fumigatus aislados mediante lavado broncoalveolar (Figura 2).

Los pacientes con cáncer pulmonar represen$\tan$ a 14 de los 187 pacientes de la serie $(7,5 \%)$. En el análisis de los pacientes con infecciones respiratorias, representan el 30\% (10 pacientes) y 46\% incluyendo sólo a los con cáncer de órgano sólido. De ellos, 9 pacientes ingresaron con foco respiratorio bajo ( 8 con neumonía, uno con bronquitis). Ninguno cursó con neutropenia febril. Los microorganismos aislados en los pacientes con neumonía fueron: $S$. pneumoniae en hemocultivos en un caso y $P$. jirovecii mediante técnica de PCR en gárgaras en dos casos. Tres de los 10 pacientes requirieron traslado a Unidad de Cuidados Intensivos (UCI), 2 de los cuales fallecieron durante su estadía hospitalaria.

En el seguimiento clínico en el hospital, 8 pacientes $(24 \%)$ se agravaron (6 con neumonía y 1 con empiema) requiriendo traslado a UPC, con una mortalidad de 50\% (Tabla 3) La mortalidad en el hospital por neumonía fue $22 \%$ (4 de 18 ) y la mortalidad general fue $12 \%$ (4 de 33 , todos con neumonía).
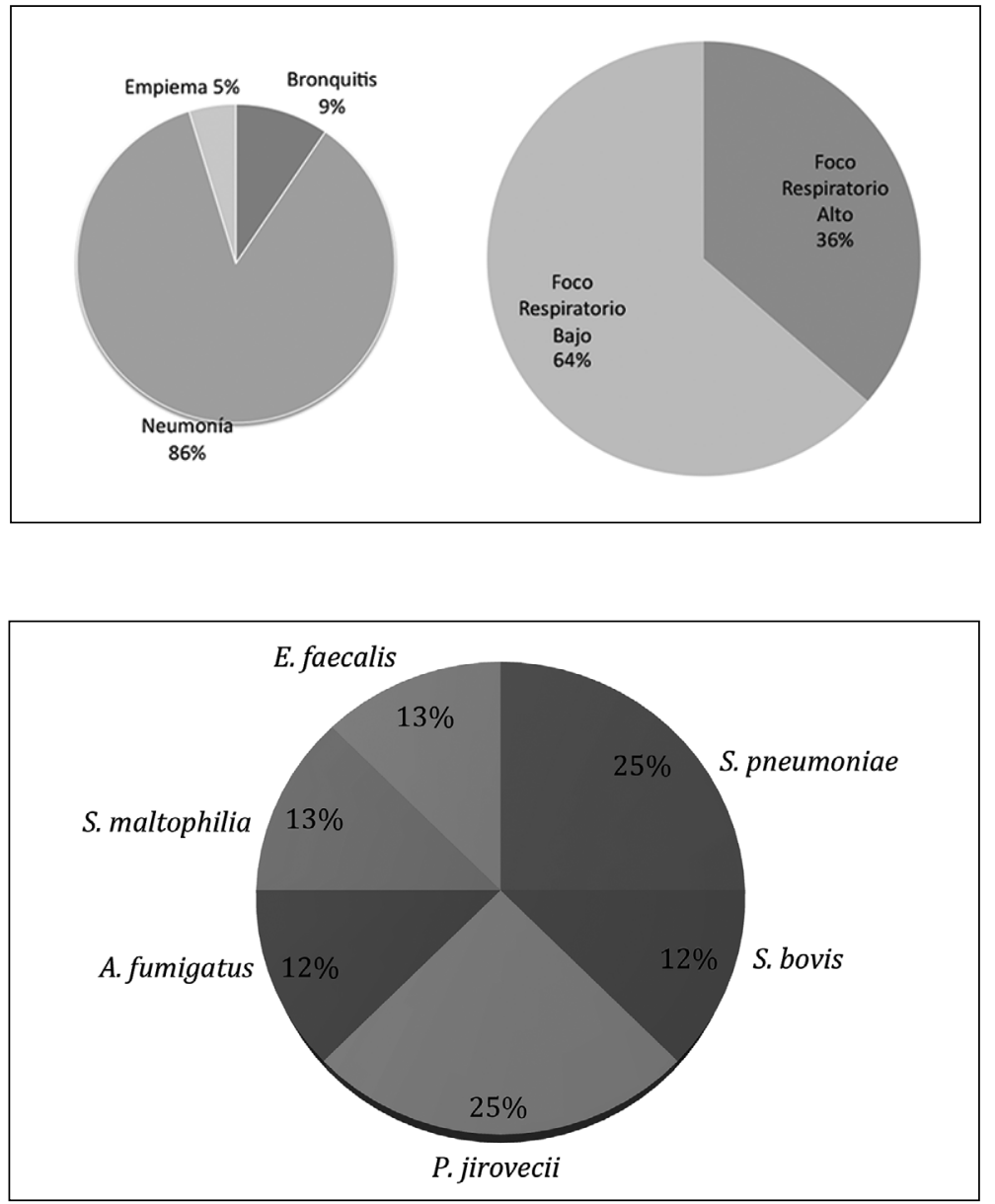

Figura 1. Infecciones respiratorias altas y bajas en pacientes adultos con cáncer hospitalizados en el Hospital Clínico de la Pontificia Universidad Católica de Chile.
Figura 2. Etiología de la neumonía en pacientes con cáncer. 
Tabla 3. Pacientes que presentan agravamiento de condición

\begin{tabular}{|lcccccc|}
\hline Patología oncológica & Edad & Género & Diagnóstico & $\begin{array}{c}\text { Lugar ingreso } \\
\text { inicial }\end{array}$ & $\begin{array}{c}\text { Etiología } \\
\text { Condición } \\
\text { egreso }\end{array}$ \\
Linfoma Hodgkin & 89 & Masculino & Neumonía & Sala & No & Fallece \\
Cáncer pulmonar & 53 & Masculino & Neumonía & Sala & No & Vivo \\
Cáncer pulmonar & 55 & Masculino & Neumonía & Sala & No & Fallece \\
Cáncer pulmonar & 53 & Masculino & Neumonía & Sala & No & Fallece \\
Carcinoma nasofaríngeo & 49 & Masculino & Respiratorio alto & Sala & No & Vivo \\
Linfoma no Hodgkin & 72 & Femenino & Neumonía & Sala & No & Fallece \\
Mieloma múltiple & 60 & Femenino & Empiema & Sala & S. aureus & Vivo \\
Mesotelioma & 43 & Femenino & Neumonía & UPC & No & Vivo \\
\hline
\end{tabular}

\section{Comentarios}

El riesgo de infección en pacientes con enfermedad neoplásica depende de la integridad de los mecanismos de defensa del huésped, incluidas las barreras anatómicas, la inmunidad celular/humoral, y la intensidad de la exposición a microorganismos potencialmente patógenos? ${ }^{7}$. El daño a las barreras anatómicas es común después de la quimioterapia, radioterapia o procedimientos invasivos, como instalación de catéteres y procedimientos quirúrgicos. La patología oncológica en sí misma, como también las intervenciones terapéuticas inmunosupresoras (quimioterapia), los corticoides y la radioterapia son responsables de los defectos inmunes en nuestros pacientes. El estado de salud del paciente, su estado funcional y comorbilidad son otros factores importantes, por ejemplo enfermedad pulmonar obstructiva crónica, diabetes mellitus, insuficiencia cardiaca, daño hepático o renal crónico representan factores de riesgo adicionales. Por último, las estadías hospitalarias frecuentes conducen a cambios de la microflora endógena y colonización con microorganismos potencialmente patógenos, que en combinación con las alteraciones determinadas por la neoplasia, facilitan la proliferación de microorganismos, ocasionando un aumento de la morbilidad y la mortalidad ${ }^{8-9}$.

En el presente estudio, logramos caracterizar el perfil epidemiológico y clínico de las infecciones respiratorias en pacientes adultos hospitalizados con cáncer hematológico o de órganos sólidos en tratamiento multimodal atendidos en nuestro centro. A nivel global, destaca el alto porcentaje de identificación clínica del foco infeccioso en la serie, dentro de las cuales las infecciones respiratorias tanto altas como bajas ocupan el segundo lugar, luego del foco gastrointestinal. De todos modos debe tenerse en cuenta la dificultad que puede existir en identificarlo clínicamente, particularmente en pacientes neutropénicos, donde pueden interponerse los síntomas respiratorios altos con la mucositis ${ }^{4}$. Sin embargo, nos parece importante insistir en la búsqueda e identificación del foco clínico con el objeto de emplear el esquema antimicrobiano con cobertura específica para el foco identificado 5 .

Dentro de las infecciones respiratorias en los pacientes oncológicos, la más importante en magnitud y pronóstico es la neumonía, representando el $75 \%$ de los pacientes que se agravaron y requirieron traslado a UPC, asociado a una muy alta mortalidad. Estrategias preventivas como la evaluación de la deglución en pacientes adultos mayores y los programas de vacunación (neumococo e influenza) son estrategias recomendadas en las guías clínicas que debemos considerar al evaluar a estos pacientes ${ }^{10}$.

Si bien no se identificaron virus en esta serie, su estudio sistemático en pacientes oncológicos con síntomas respiratorios es fundamental, principalmente en períodos de alta circulación ${ }^{11}$. Cabe destacar que el examen virológico utilizado en este estudio correspondió a la técnica de inmunofluorescencia directa. Sin embargo, actualmente en nuestro centro se dispone del panel viral molecular por técnica de biología molecular, el cual debe ser evaluado dirigidamente en este tipo de pacientes en los próximos estudios.

En nuestra serie destaca la alta prevalencia de pacientes con cáncer pulmonar con infecciones respiratorias, donde este foco es el principal motivo de ingreso $(71 \%)$ tal como se ha descrito en otras series ${ }^{9}$, debiendo incluir en el diagnóstico diferencial el tromboembolismo pulmonar, reacción adversa a drogas o incluso fiebre tumoral. Dos pacientes presentaron neumonía por $P$. 
jirovecii, aislado mediante reacción en cadena de polimerasa en gárgaras. Sin embargo, atribuir la etiología a este germen es motivo de discusión, dado que en población sana puede llegar a encontrarse en más del $60 \%$ de los casos ${ }^{12}$.

El manejo de las infecciones es también parte de la lucha contra el cáncer, ya que alteran el curso del tratamiento oncológico, posponiendo quimioterapias y afectan la sobrevida global. Un enfrentamiento precoz, basados en el seguimiento de la curva febril y el alza de los parámetros inflamatorios (principalmente la PCR sérica) debe orientar al clínico en la búsqueda sistemática del foco infeccioso y la etiología microbiológica, evitando el retraso en el inicio de la cobertura antimicrobiana apropiada, incluyendo a los microorganismos oportunistas.

Las principales limitaciones de este estudio están determinadas por la falta de registro del performance status del paciente, como también el estadio clínico, comorbilidades y detalles del tratamiento de la patología oncológica. Por otro lado, el estudio microbiológico y radiológico no se ciñó a un protocolo. El pequeño tamaño muestral, además, no permite realizar un análisis estadístico más detallado. Sin embargo, serán tomados en consideración estas variables en las próximas series para intentar caracterizar mejor la patología respiratoria en los pacientes oncológicos atendidos en nuestra red de salud.

Si bien este estudio se refiere sólo a la experiencia de un centro, demuestra la importancia de la vigilancia de las infecciones respiratorias en pacientes oncológicos para definir conductas locales; la generalización de estos datos no es posible, pero pueden ser orientadores para centros que atiendan pacientes adultos con patologías similares.

En conclusión, queda establecido el perfil epidemiológico de los pacientes oncológicos hospitalizados con infecciones respiratorias agudas, destacando la neumonía, que conlleva una elevada morbimortalidad y etiología microbiana variada, que nos permite orientarnos a las conductas antimicrobianas empíricas iniciales.

\section{Abreviaciones}

HCUC: Hospital Clínico de la Pontificia Universidad Católica de Chile.

RAN: Recuento absoluto de neutrófilos.

PCR: Proteína $\mathrm{C}$ reactiva.

FOD: Fiebre de origen desconocido.

SCoN: Staphylococcus coagulasa negativa.

Score de MASCC: Multinational Association for Supportive Care in Cancer Risk Index.

\section{Bibliografía}

1.- VELASCO E, THULER L C S, MARTINS C A S, DIAS L M C, GONÇALVES V M S. Risk factors for bloodstream infections at a cancer center. Eur J Clin Microbiol Infect Dis 1998; 17: 587-90.

2.- ELTING L S, RUBENSTEIN E B, ROLSTON K V I, BODEY G P. Outcomes of bacteremia in patients with cancer and neutropenia: observations from two decades of epidemiological and clinical trials. Clin Infect Dis 1997; 25: 247-59.

3.- VELASCO E, BYINGTON R, MARTINS C S A, SCHIRMER M, DIAS L C M, GONÇALVES V M S C. Bloodstream infection surveillance in a cancer centre: a prospective look at clinical microbiology aspects. Clin Microbiol Infect 2004; 10: 542-9.

4.- RABAGLiATI R, FUENTES G, ORELLANA E, OPORTO J, DOMÍNGUEZ I, BENÍTEZ R, et al. Etiología de episodios de neutropenia febril en pacientes adultos con cáncer hematológico y de órganos sólidos en el Hospital Clínico Universidad Católica, SantiagoChile. Rev Chil Infect 2009; 26: 106-13.

5.- SANTOLAYA M E, RABAGLIATI R, BIDART T, PAYÁ E, GUZMÁN A M, MORALES R, et al. Consenso manejo racional del paciente con cáncer, neutropenia y fiebre. Rev Chil Infect 2005; 22 (Supl 2): S79.

6.- GARCÍA P, BENÍTEZ R, LAM M, SALINAS A M, WIRTH H, ESPINOZA C, et al. Coagulase negative staphylococci: clinical, microbiological and molecular features to predict true bacteraemia. J Med Microbiol 2004; 53: 67-72.

7.- MCDONALD F, ATKINS C. Defective cytostatic activity of pulmonary alveolar macrophages in primary lung cancer. Chest 1990; 98: 881-5.

8.- BODEY P. Infection in cancer patients. A continuing association. Am J Med 1986; 81: 11-26.

9.- AKINOSOGLOU K S, KARKOULIAS K, MARANGOS M. Infectious complications in patients with lung cancer. Eur Rev Med Pharmacol Sci 2013; 17: 8-18.

10.- NATIONAL CENTER FOR IMMUNIZATION AND RESPIRATORY DISEASES. General recommendations on immunization-recommendations of the Advisory Committee on Immunization Practices (ACIP). MMWR Recomm Rep 2011; 60: 1.

11.- DÍAZ A, BARRÍA P, NIEDERMAN M, RESTREPO M, DREYSE J, FUENTES G, et al. Etiology of community-acquired pneumonia in hospitalized patients in Chile: The increasing prevalence of respiratory viruses among classic pathogens. Chest 2007; 131: 779-87.

12.- PONCE C, GALLO M, BUSTAMANTE R, VARGAS S. Pneumocystis colonization is highly prevalent in the autopsied lungs of the general population. Clin Infect Dis 2010; 50: 347-53.

Correspondencia a:

Dr. Gino Fuentes L.

Departamento Enfermedades Respiratorias, Facultad de Medicina, Pontificia Universidad Católica de Chile. Marcoleta 350. Santiago, Chile.

Email: gpfuente@uc.cl 